\title{
Mapeamento prospectivo científico e tecnológico da família Anacardiaceae R. Br.
}

\author{
Prospective scientific and technological mapping of the family Anacardiaceae R. Br. \\ Cartografía científica y tecnológica prospectiva de la familia Anacardiaceae R. Br.
}

Davi Nascimento Costa

ORCID: https://orcid.org/0000-0002-1900-1754 Universidade Federal do Delta do Parnaíba, Brasil E-mail: daveoficial123@gmail.com

Ruanna Thaimires Brandão Souza ORCID: https://orcid.org/0000-0002-9416-8327 Universidade Federal do Piauí, Brasil E-mail: ruanna_na15@hotmail.com

Regigláucia Rodrigues de Oliveira ORCID: https://orcid.org/0000-0003-3916-3686 Universidade Federal do Piauí, Brasil E-mail: regiglaucia@hotmail.com

Renata Brito dos Reis

ORCID: https://orcid.org/0000-0003-4595-7810 Universidade Federal do Delta do Parnaíba, Brasil E-mail: renata_britoreis@hotmail.com

Maria Gracelia Paiva Nascimento ORCID: https://orcid.org/0000-0001-9652-5730 Universidade Federal do Piauí, Brasil

E-mail: graceliapaiva@gmail.com

Giovanna Santos de Souza

ORCID: https://orcid.org/0000-0002-4982-0988 Universidade Federal do Piauí, Brasil E-mail: gih.ssouza@gmail.com

Maria do Amparo de Moura Macêdo ORCID: https://orcid.org/0000-0001-5176-0112 Universidade Federal do Piauí, Brasil E-mail: amparo_macedo@hotmail.com

Nailton de Souza Araujo ORCID: https://orcid.org/0000-0002-7529-5750 Universidade Federal do Piauí, Brasil

E-mail: nailtonbio4@gmail.com

Emerson Bruno Castro Mesquita ORCID: https://orcid.org/0000-0003-0914-226X Universidade Federal do Delta do Parnaíba, Brasil E-mail: ebcmesquita@hotmail.com

Giovana Sarah Sales Batista

ORCID: https://orcid.org/0000-0003-2121-6399

Universidade Federal do Delta do Parnaíba, Brasil E-mail: saarasales.b@gmail.com

Ivanilza Moreira de Andrade

ORCID: https://orcid.org/0000-0001-6059-8540

Universidade Federal do Delta do Parnaíba, Brasil

E-mail: ivanilzaandrade@hotmail.com

\begin{abstract}
Resumo
Anarcadiaceae é uma das famílias de angiospermas que apresenta expressiva diversidade no Brasil, além de importância econômica e medicinal. Objetivou-se realizar uma prospecção tecnológica e científica sobre Anacardiaceae, analisando o número de depósitos de patentes por países, ano e suas classificações internacionais (CIP), bem como descrever o perfil da produção científica nas áreas de aplicação. Realizou-se uma prospecção científica e tecnológica em bases de dados de artigos e patentes, nacional e internacional, sobre Anacardiaceae. Os pedidos de depósito de patentes tiveram início em 2012 com um aumento de publicações em 2015 e 2020 de acordo com Web of Science e Scopus, respectivamente. O Japão, Estados Unidos e China lideram com a produção tecnológica incluída, majoritariamente na subclasse $\mathrm{A} 61 \mathrm{~K}$, e as tecnologias são direcionadas, principalmente, para finalidades médicas, odontológicas ou higiênicas. Já na produção científica, o Brasil se destaca em primeiro lugar em número de artigos publicados. Conclui-se que a família é fonte de diversas pesquisas médicas, e o Brasil apresenta grande potencial biotecnológico com a utilização desta.
\end{abstract}

Palavras-chave: Botânica; Patentes; Produção científica. 


\begin{abstract}
Anarcadiaceae is one of the families of angiosperms that presents an expressive diversity in Brazil, besides its economic and medicinal importance. The objective was to perform a technological and scientific prospection about Anacardiaceae, analyzing the number of patent deposits per country, year and their international classifications (CIP), as well as to describe the profile of the scientific production in the areas of application. A scientific and technological prospection was carried out in national and international databases of articles and patents on Anacardiaceae. Patent applications started in 2012 with an increase in publications in 2015 and 2020 according to Web of Science and Scopus, respectively. Japan, the United States, and China lead with technological production included mostly in subclass A61K, and the technologies are directed mainly to medical, dental, or hygienic purposes. As for scientific production, Brazil stands out in first place in number of published articles. We conclude that the family is the source of several medical researches, and Brazil has a great biotechnological potential with its use.
\end{abstract}

Keywords: Botany; Patents; Scientific production.

\title{
Resumen
}

Las anarcadiáceas son una de las familias de angiospermas que presentan una expresiva diversidad en Brasil, además de su importancia económica y medicinal. El objetivo fue realizar una prospección tecnológica y científica sobre las Anacardiáceas, analizando el número de depósitos de patentes por países, año y sus clasificaciones internacionales (CIP), así como describir el perfil de la producción científica en las áreas de aplicación. Se realizó una prospección científica y tecnológica en bases de datos nacionales e internacionales de artículos y patentes sobre Anacardiaceae. La presentación de solicitudes de patentes comenzó en 2012 con un aumento de las publicaciones en 2015 y 2020 , según Web of Science y Scopus, respectivamente. Japón, Estados Unidos y China están a la cabeza de la producción tecnológica, en su mayoría incluida en la subclase A61K, y las tecnologías se dirigen principalmente a fines médicos, dentales o higiénicos. En cuanto a la producción científica, Brasil destaca en primer lugar en número de artículos publicados. Se concluye que la familia es una fuente de varias investigaciones médicas, y Brasil presenta un gran potencial biotecnológico con su uso.

Palabras clave: Botánica; Patentes; Producción científica.

\section{Introdução}

Anacardiaceae R. Br. está representada por 81 gêneros e 800 espécies, ocorrendo tanto em habitat secos como úmidos, mais frequentemente em terras baixas, encontradas em regiões tropicais e subtropicais por todo globo, presente ainda em regiões temperadas (Pell, 2011). No Brasil ocorrem 15 gêneros e 64 espécies, das quais 14 ocorrem exclusivamente no país (Flora do Brasil, 2020a).

Espécies de Anacardiaceae caracterizam-se por árvores, arbustos, raro subarbustos ou lianas, inermes ou raramente armados, aromáticas, canais resiníferos bem desenvolvidos principalmente na casca e no floema de pecíolos e nervuras calibrosas das folhas, além de presentes em flores, frutos e outros tecidos. Folhas alternas, raro opostas ou verticiladas, geralmente imparipinadas, às vezes trifoliadas ou simples, sésseis ou pecioladas, sem estípulas; folíolos alternos, opostos ou subopostos, margem inteira, serrada, dentada, crenada, crenado-serrada ou erosa, venação pinada. Inflorescências axilares ou terminais, panículas, racemos, pseudorracemos, tirsoides, raro, flores solitárias; pedicelos articulados ou não, brácteas e bractéolas decíduas ou persistentes. Flores unissexuais e/ou bissexuais (plantas dioicas, monoicas, andromonoicas, polígamas ou hermafroditas), diclamídeas, actinomorfas, hipóginas. Frutos geralmente drupas, às vezes sâmaras ou bagas, carnoso ou seco, alado ou não, às vezes com cálice cartáceo acrescente ou com hipocarpo carnoso; mesocarpo às vezes com canais resiníferos negros proeminentes. Sementes 1-4(-12), endosperma escasso ou ausente; embrião curvo ou reto; cotilédones geralmente plano-convexos ou achatados e distintos, geralmente do mesmo tamanho (Flora do Brasil, 2020b).

A família tem grande importância econômica, devido muitas espécies fornecerem frutos comestíveis, madeiras úteis ou serem de usos ornamentais. Como exemplo, a semente do cajueiro (Anacardium occidentale L.) obtém-se a castanha-decaju, enquanto o pedicelo frutífero suculento (hipocarpo) é comercializado in natura (Araújo et al., 2018; Santos et al., 2018; Nunes et al., 2018). Apresenta também compostos fenólicos e propriedades medicinais (Santos et al., 2018). Outros frutos de importância comercial ou regional dessa família incluem a manga (Mangifera indica L.), o cajá (Spondias mombin L.), o umbu (Spondias tuberosa Arruda) e a seriguela (Spondias purpurea L.). Schinus terebinthifolia Raddi, S. molle L. e Rhus 
succedanea $\mathrm{L}$. são exemplos de plantas utilizadas na ornamentação de ruas e praças.

Entre as espécies que apresentam madeira de boa qualidade estão o gonçalo-alves (Astronium fraxinifolium Schott ex Spreng.), o guaritá (A. graveolens Jacq.), a aroeira (A. urundeuva (M. Allemão) Engl.), a aroeira-branca (Lithraea molleoides (Vell.) Engl.) e a braúna (Schinopsis brasiliensis Engl.) (Souza \& Lorenzi, 2005).

Algumas atividades biológicas já foram identificadas em Anacardiaceae, como propriedades antioxidante (Da Silva et al., 2015), atividades inibidoras da acetilcolinesterase e indutora de quinona redutase, que se torna importante para avaliar a capacidade da indução da quimioproteção contra eventos da carcinogênese (Zeraik et al., 2016). Substâncias bioativas também foram identificadas no grupo, como compostos fenólicos e flavonoides, bem como substâncias já conhecidas previamente, como o ácido gálico e isotachioside, e as substâncias inédias 3,4-dihidroxifeniletanol-5- $\beta$-D-glicose e 5- hydroxyl-4metoxibenzoico-3-0-p-Dglicose benzóico (Uchôa et al., 2015).

Neste sentido, estudos são realizados para encontrar e validar o potencial curativo de plantas, para diferentes patologias. Isto torna-se relevante, pois comprovada a eficácia medicinal, se possa investir em tecnologia e disponibilizar os produtos para a sociedade. Por isto, faz-se necessária a investigação científica com plantas medicinais para obtenção de medicamentos e outros produtos naturais, que possam ser patenteados como novas tecnologias na indústria (Berlinck et al., 2017).

Além disto, considerando as contribuições da investigação científica e tecnológica com vegetais, depreende-se que o estudo de plantas medicinais se torna um importante nicho econômico, uma vez que as espécies brasileiras representam grande eficácia medicinal, além do país possuir a maior cobertura vegetal do mundo (Da Silva Oliveira et al., 2020).

Destaca-se que as espécies conhecidas popularmente como pau-ferro (Myracrodruon balansae (Engl.) Santin) e Quebracho (Schinopsis balansae Engl.) estão listadas como ameaçadas de extinção para o táxon no Brasil, isto deve-se a atividades agropecuárias e, principalmente, pelas monoculturas de espécies exóticas e ameaças de queimadas e ação antrópica (Martinelli \& Moraes, 2013).

Diante do exposto objetivou-se realizar uma prospecção tecnológica e científica sobre Anacardiaceae, analisando o número de depósitos de patentes por países, ano e suas classificações internacionais (CIP), bem como descrever o perfil da produção científica nas áreas de aplicação.

\section{Metodologia}

Esta pesquisa é do tipo documental exploratória de abordagem quantitativa (De Carvalho et al., 2020). A busca dos dados foi realizada em agosto de 2021, utilizando como descritor o nome da família "Anacardiaceae" e mais três descritores combinados com o operador booleano AND da seguinte maneira: "Anacardiaceae AND Taxonomy", "Anacardiaceae AND Food" e "Anacardiaceae AND Production" (Figura 1) nos bancos de artigos e patentes publicadas de 1940 a 2021, sendo considerados válidos os documentos que apresentassem esses termos no título e/ou resumo.

Para realizar a prospecção tecnológica foram consultados os seguintes bancos de patentes: World Intellectual Property Organizacion (WIPO) e Instituto Nacional da Propriedade Industrial (INPI) e Classificação Internacional de Patente (CIP). Quando se realiza prospecção tecnológica, sabe-se que a Classificação Internacional de Patentes (CIP), distribui as patentes em classificações de acordo com suas aplicações. Sua estrutura de classificações segue uma ordem hierárquica dividida em oito seções, 21 subseç̃oes, 120 classes, 628 subclasses e 69.000 grupos (Serafini et al., 2012).

A prospecção científica foi obtida nas bases de artigos e resumos Web of Science (WoS) e Scopus, os documentos foram analisados quanto ao ano, países e áreas de publicação. A opção pela consulta nas respectivas bases de artigos e patentes se deu pela confiabilidade e versatilidade dos dados indexados principalmente para artigos científicos dado o elevado volume de publicações disponíveis. 
Os dados foram tabulados de forma individual e organizados em tabelas e gráficos no Microsoft Excel (2019), visando analisar os dados e explana-los por meio de gráficos, esquemas, mapas e tabelas.

Figura 1. Fluxograma com as etapas da pesquisa.

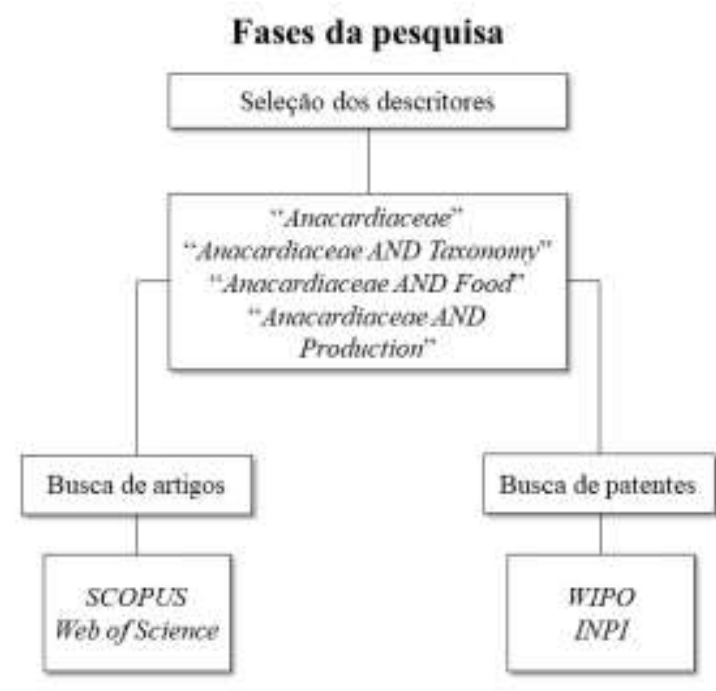

Fonte: Autores (2021).

\section{Resultados e Discussão}

Utilizando as bases de artigos Web of Science e Scopus, com emprego do descritor "Anacadiaceae", obteve-se o total de 2268 e 3394 artigos indexados, respectivamente. No entanto, com o objetivo de refinar os dados utilizou-se uma busca com os descritores “Anacardiaceae AND Taxonomy", e a quantidade de trabalhos indexados correspondeu a 89 e 144 , respectivamente. Utilizando os descritores “Anacardiacaeae AND Food” encontrou-se 259 e 290 pesquisas científicas nas bases Scopus e Web of Science. Com as palavras-chave “Anacardiaceae AND Production” registraram-se 303 e 272, manuscritos respectivamente indexados nas bases internacionais Scopus e Web of Science.

Para a busca de patentes e áreas tecnológicas, foram registrados para o descritor Anacardiaceae 112 e 1 nas bases WIPO e INPI, respectivamente, e nenhum registro para os descritores "Anacardiaceae AND Taxonomy". Utilizando "Ancardiaceae AND Food" foram encontradas no banco de dados da WIPO 1650 patentes indexadas. Os descritores “Anacardiaceae AND Production” obteve-se o valor expressivo de patentes na base WIPO 2981 (Tabela 1).

Tabela 1. Número de artigos e patentes registrados por base de dados envolvendo os termos utilizados

\begin{tabular}{l|c|c|c|c|c}
\hline Descritores & Web of Science & Scopus & WIPO & INPI & . \\
\hline Anacardiaceae & 2268 & 3394 & 112 & 1 & \\
\hline Anacardiaceae AND Taxonomy & 89 & 144 & - & - & \\
\hline Anacardiaceae AND Food & 259 & 290 & 1650 & - & \\
\hline Anacardiaceae AND Production & 303 & 272 & 2981 & - & \\
\hline
\end{tabular}

Fonte: Autores (2021). 


\subsection{Prospecção Tecnológica}

Com base nos depósitos de patente é possível analisar que Japão e Estados Unidos da América destacam-se como maiores detentores de pedidos de patentes registradas envolvendo a família, com 17 e 13 respectivamente (Figura 2). O Japão destaca-se como um dos países que mais promovem inovações tecnológicas no mundo estando entre os 20 primeiros colocados em ranking divulgado pela Organização Mundial da Propriedade Intelectual (OMPI) (OMS, 2018). A China vem aumentando o investimento em ciência, inovação e tecnologia o que a torna uma das maiores potências mundiais em criação tecnológica e patenteamento de novos produtos (Monte, Lopes \& Contini, 2017).

Figura 2. Número de depósitos de patentes por país obtido, utilizando-se como descritor Anacardiaceae, nos bancos de dados World Intellectual Property Organization (WIPO) e Instituto Nacional de Propriedade Intelectual (INPI).

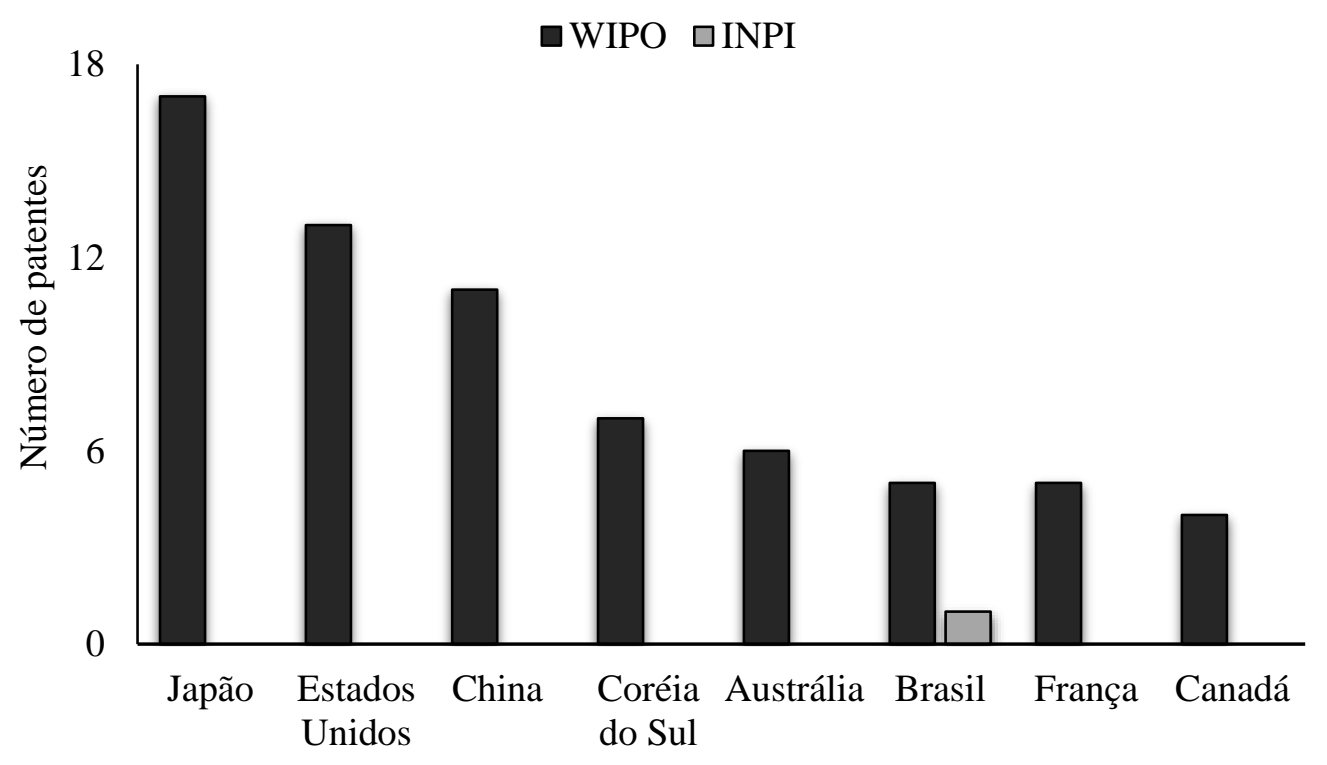

Fonte: Autores (2021).

O Brasil possui a maior biodiversidade vegetal do planeta e é considerado como um centro de diversidade do grupo, o país apresenta grande potencial inovador na área de produtos naturais. Considera-se que a biodiversidade brasileira é considerada fonte natural sofisticada de estruturas químicas a qual apresenta grandes potencialidades para a produção de inovações em vários setores, como farmacêutico, cosmético, agroquímico e alimentício (Da Silva Oliveira et al., 2020).

Devido a entraves como por exemplo as normas de regulamentação de proteção ao patrimônio genético, há um aproveitamento inadequado da diversidade vegetal do Brasil, o que tem provocado dificuldades sobre a diversidade biológica e química (Pimentel et al., 2015; Bolzani, 2016). Os dados apontam que o país pode concorrer com nações que apresentam um histórico de inovação como Japão, Estados Unidos e China, caso invista em produção e inovação tecnológica.

O número de depósitos de patentes nos bancos de dados do WIPO e INPI obtidos com o descritor Anacardiaceae no período de 2012-2021 está ilustrado na figura 3, onde é possível analisar que as publicações de patentes no banco WIPO tiveram início em $2012(n=4)$ e este valor manteve-se estável no ano seguinte. Nos últimos 9 anos, observou-se que, o maior número de tecnologia foi depositado entre 2014 e 2019 (n=6), tendo uma baixa em 2016 (n=3) (Figura 3).

No banco do INPI (Instituto Nacional de Propriedade Industral), registrou-se apenas uma patente no ano de 2017. A tecnologia tem como título "Preparação farmacêutica contendo extratos de Schinopsis brasiliensis (Anacadiaceae)", este estudo trata da utilização do extrato bruto obtido de casca desta espécie em preparações farmacêuticas com finalidade fotoprotetora e antioxidante (INPI, 2021). 
Figura 3. Evolução anual dos pedidos de depósito de patentes indexadas nos bancos de dados World Intellectual Property Organization (WIPO) e Instituto Nacional de Propriedade Industrial (INPI) utilizando o descritor Anacardiaceae.

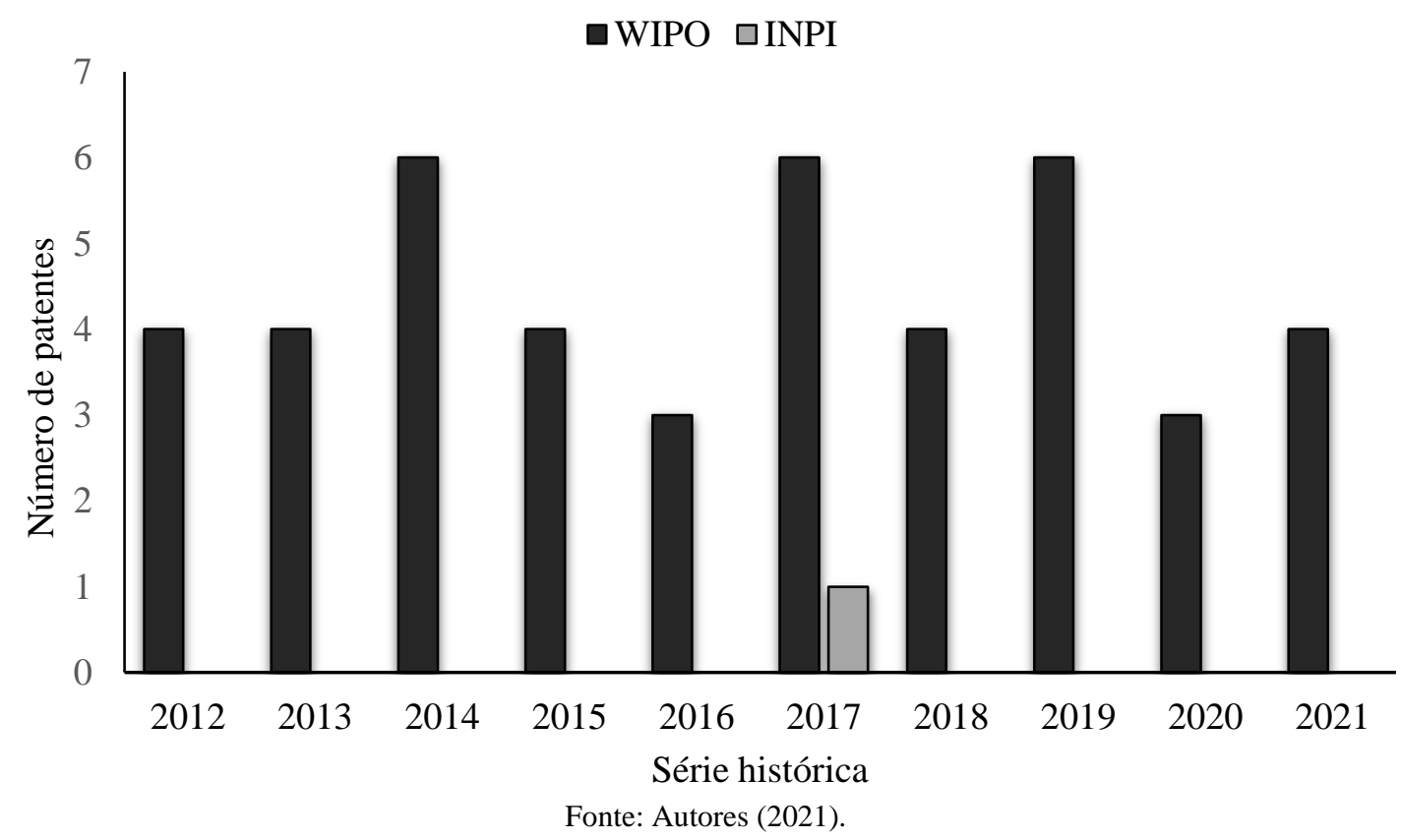

Os detentores da patente registrada no INPI destacam que Schinopsis brasiliensis, apresenta atividade antimicrobiana e que esta atividade está relacionada à concentração de compostos fenólicos no extrato, principalmente taninos, que no extrato correspondem $55 \%$ do conteúdo fenólico total. Ainda de acordo com os autores a espécie apresenta atividade antibacteriana para as cepas patógenas Staphylococcus aureus e Pseudomonas aerugionosa (Saraiva et al., 2011).

Entre os pedidos de depósitos de patentes registrados na base internacional da WIPO, 78 estão alocados na subclasse A61K (Figura 4), que trata de preparações para finalidades médicas, odontológicas ou higiênicas. Além disto, destaca-se um número expressivo de patentes (41, no total) que está relacionado à atividade terapêutica específica de compostos químicos ou preparações medicinais. Outras patentes estão alocadas em menor número em outras subclasses como A61Q (12 patentes), que aborda compostos com uso específico de cosméticos ou preparações similares para higiene pessoal. 
Figura 4. Distribuição por CIP pedidos de depósito de patentes no World Intellectual Property Organization (WIPO) e Instituto Nacional de Propriedade Industrial (INPI) utilizando Anacardiaceae.

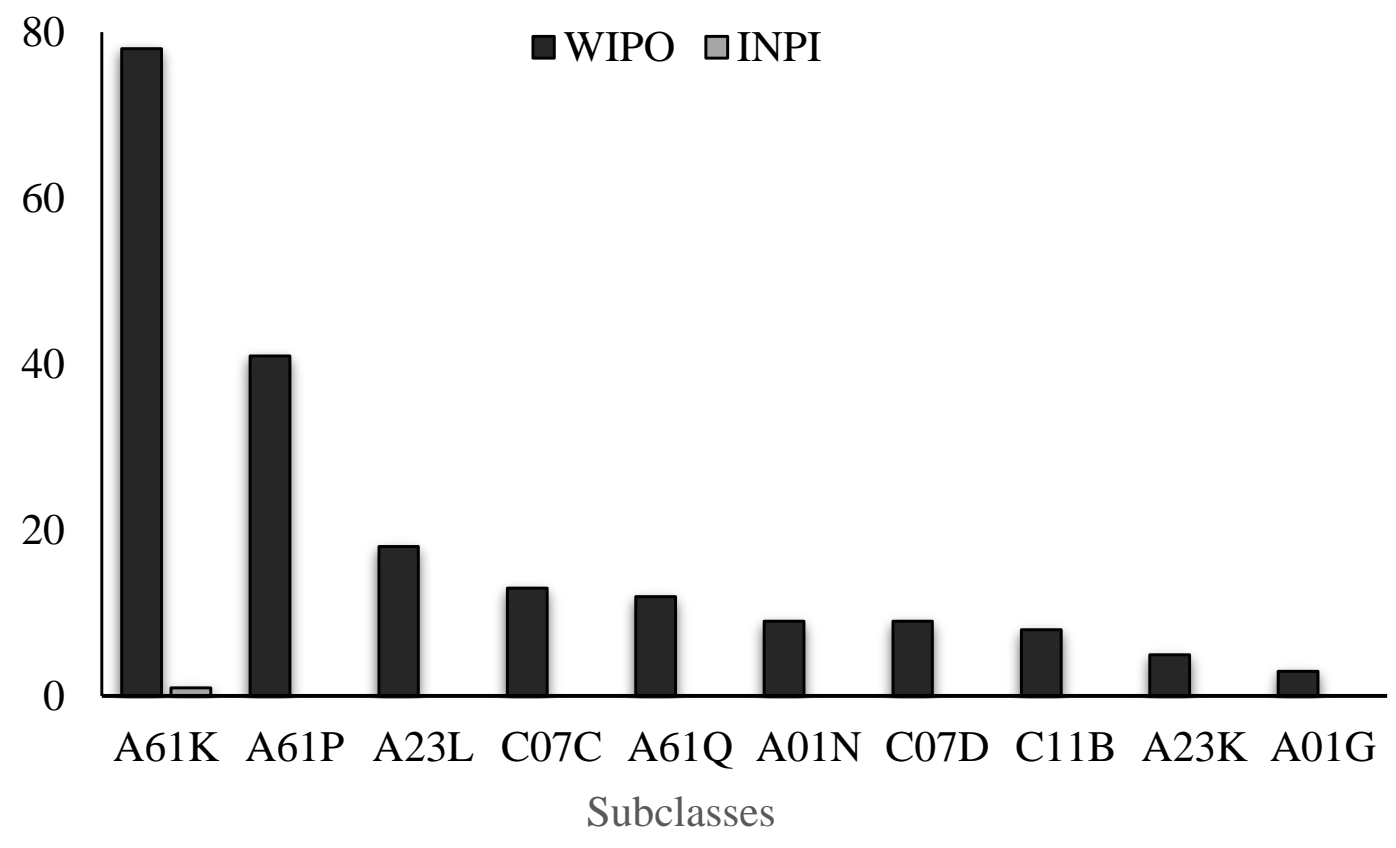

Fonte: Autores (2021).

Destaca-se a cadeia produtiva de Anacardium occidentale L. produto bastante comum em todo país de onde é retirado o insumo para fazer suco, polpa e outros produtos (Caju) (Cruz, Alencar \& Silva, 2017). Mangifera indica L. (Manga), é um produto exportado para Europa, Estados Unidos e Japão (Araújo; Campos \& Campos, 2019). Spondias mobim L. (Cajá) também é utilizada pela indústria na produção de polpas e sucos naturais (Jacobsen et al., 2018).

\subsection{Prospecção Científica}

O Brasil é o país com maior número de pesquisas cientificas que culminaram em publicações indexadas nas bases utilizadas, apresentando 731 e 490 publicações nas bases Scopus e Web of Science (Figura 5). O país registra quantidade expressiva de gêneros e espécies da família, Albuquerque et al. (2015) destacaram que a busca por compostos fenólicos, substâncias bioativas e metabólitos secundários em espécies do táxon tem sido objeto de diversas pesquisas no campo químico o que justificaria a quantidade de publicações. Além disto, a produção e valoração econômica da família também se destaca como fator importante para realização destas publicações (Fyfe et al., 2020; Lacaris et al., 2019; Oliveira et al., 2018). 
Figura 5. Distribuição por país de artigos indexados na base Scopus e Web of Science com o descritor Anacardiaceae.

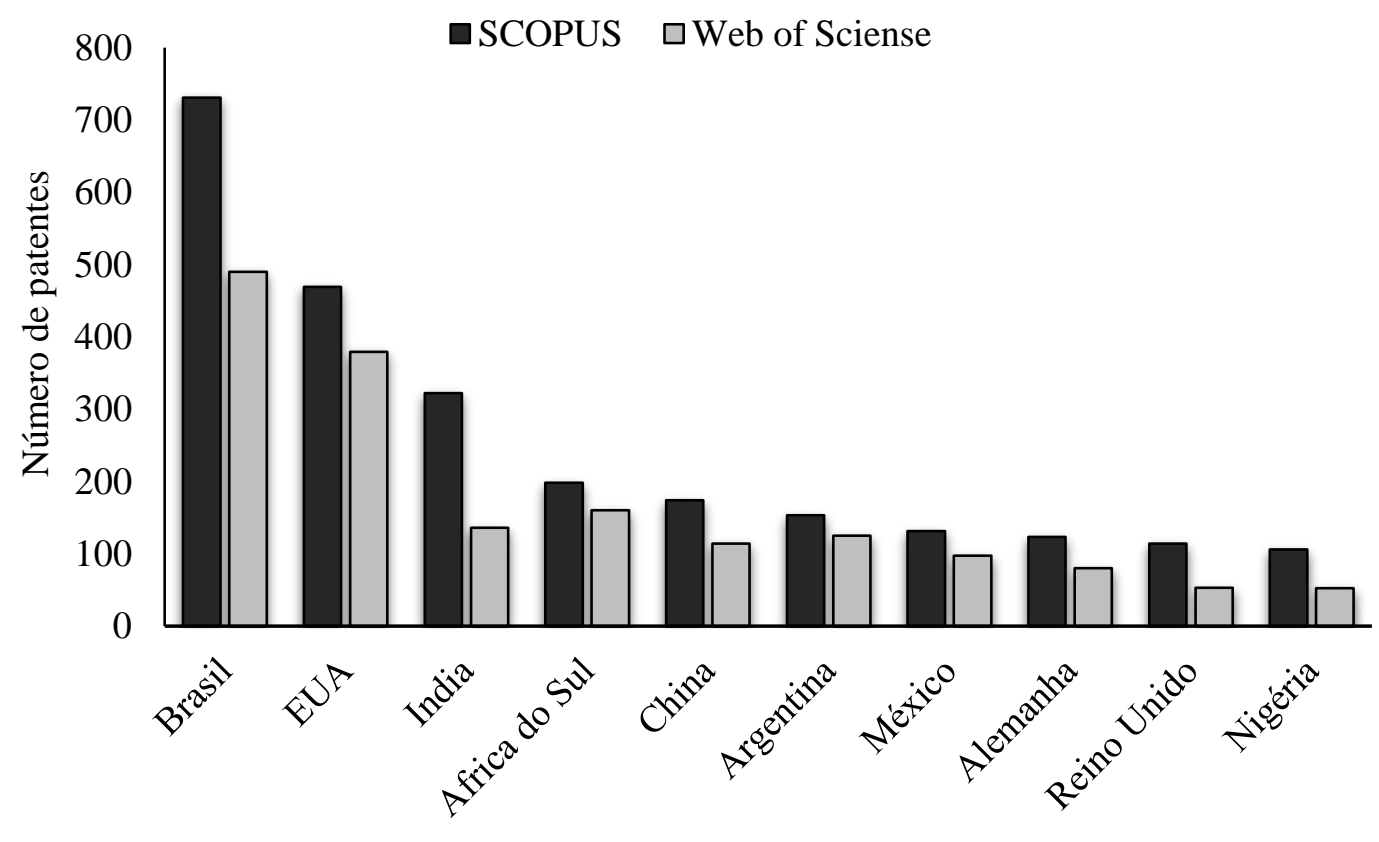

Fonte: Autores (2021).

A Figura 6 representa a evolução das publicações indexadas na base de artigos internacionais entre os anos de 19402021 para Anacardiaceae. A primeira publicação, realizada em 1940, trata sobre o látex e cristais de Schinus terebinthifolius Raddi, que ocorre no Japão (Morishige, 1940). As publicações tiveram início em 1997 na base Web of Science e o maior número de artigos indexados ocorreu em 2020 (n=149), com a maior parte destas pesquisas na área de Botânica (23,28\%), e as pesquisas incluindo descrições taxonômicas, anatômicas e pesquisas sobre diversidade genética de espécies do táxon (Tavares et al., 2020; Barrett et al., 2020; Cho, Jang \& Lee, 2020; Bordignon \& Ely, 2020).

$\mathrm{Na}$ Scopus, o maior número de artigos foi registrado em $2016(\mathrm{n}=214)$ na base de dados neste ano é possível observar que o maior número de pesquisas estão inclusas na área Agricultura e Ciências Biológicas (28,7\%), e as publicações destinamse em sua maior parte a descrever características relacionadas a diversidade morfológica, genética e filogenética de espécies de Anacardiaceae (Muellner-Riehl et al., 2016; Harsono et al., 2016; Yang et al., 2016), além da busca por caracterização química e de metabólitos secundários com espécies do táxon (Nagel et al., 2016; Li et al., 2016; Alencar et al., 2016). 
Figura 6. Evolução anual de artigos indexados na base Scopus e Web of Science com Anacardiaceae.

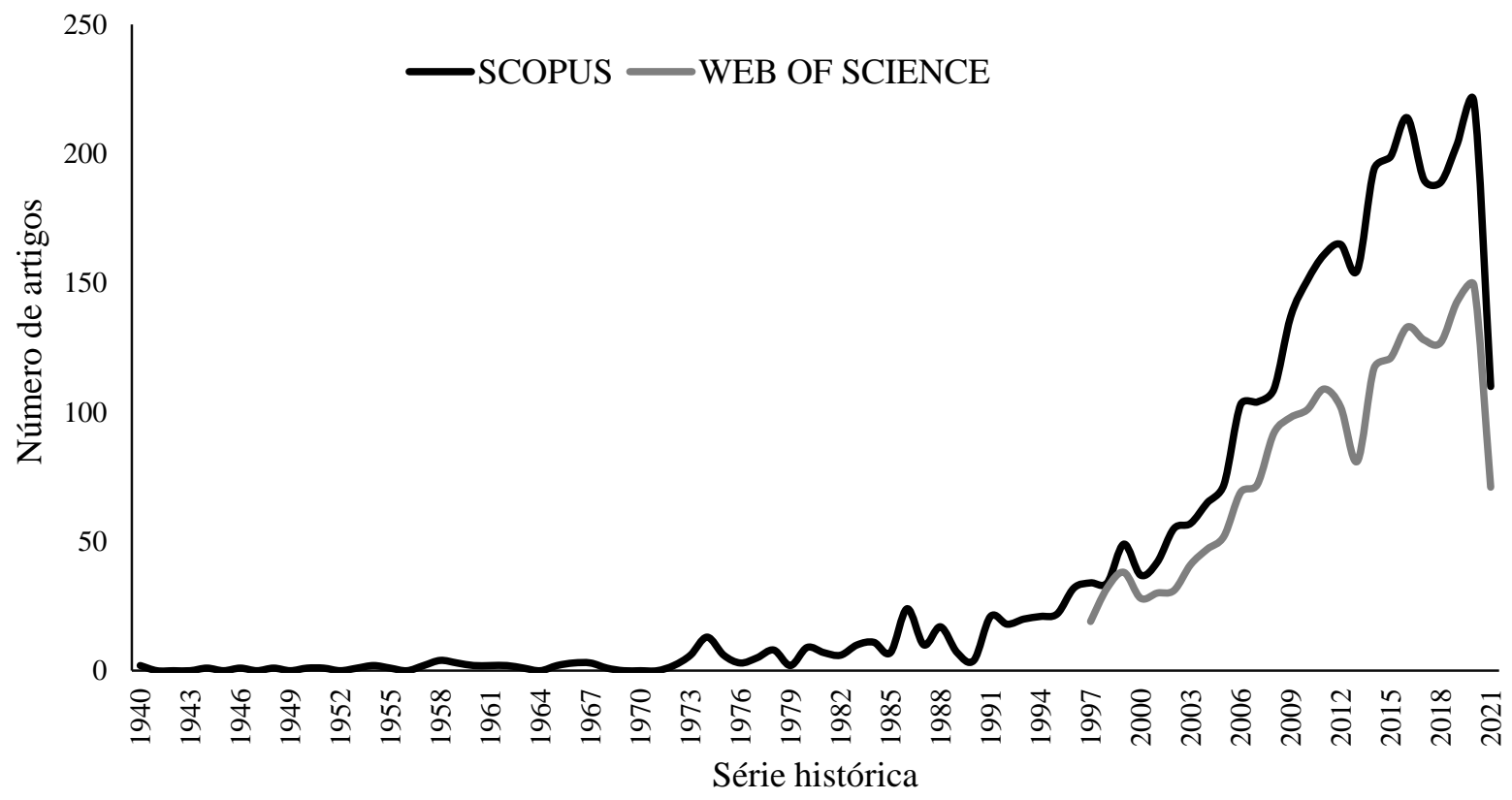

Fonte: Autores (2021).

Das áreas mais representativas quanto ao número de manuscritos indexados para o táxon, analisou-se que as áreas de Ciências Agrárias e Biológicas aparecem em primeiro lugar com 1695 (32,70\%) artigos, seguida de Farmacologia, Toxicologia e Farmacêutica com 809 (15,60\%). Bioquímica, Genética e Biologia Molecular também se encontram entre as áreas mais pesquisadas relacionando as plantas da família, com 760 (14,60\%) dos manuscritos publicados (Tabela 2).

Tabela 2. Distribuição com das publicações indexadas por áreas na base Scopus com Anacardiaceae.

\begin{tabular}{ccc}
\hline Areas de interesse & SCOPUS & $\%$ \\
\hline Ciências Agrárias e Biológicas & 1695 & $32,70 \%$ \\
\hline Farmacologia, Toxicologia e Farmacêutica & 809 & $15,60 \%$ \\
\hline Bioquímica, Genética e Biologia Molecular & 760 & $14,60 \%$ \\
\hline Medicina & 628 & $12,10 \%$ \\
\hline Química & 403 & $7,80 \%$ \\
\hline Ciências Ambientais & 264 & $5,10 \%$ \\
\hline Imunologia e Microbiologia & 138 & $2,70 \%$ \\
\hline Ciências da terra & 103 & $2 \%$ \\
\hline Engenharia Química & 71 & $1,40 \%$ \\
\hline Multidisciplinar & 65 & $1,30 \%$ \\
\hline Outras & 219 & $4,90 \%$ \\
\hline
\end{tabular}

Fonte: Autores (2021).

A concentração de trabalhos em áreas que envolvem produção de alimentos, destaca- se pela importância econômica da família, onde os frutos são utilizados na alimentação humana ou animal (Salesse et al., 2018; Gurjão et al., 2015; Oliveira, Moraes \& De Carvalho, 2017). Destaca-se também que a busca por compostos fenólicos, substâncias bioativas, propriedades medicinais e farmacêuticas torna-se um fator decisivo no volume de trabalhos voltados às áreas de Farmacologia, toxicologia e Farmacêutica, isto está relacionado ao fato de diversas espécies do táxon, possuírem propriedades antimicrobianas (Santos, 
Santos \& Marisco, 2017; Monteiro et al., 2017; Ribeiro et al., 2020), antioxidantes (Broinizi et al., 2007; Chaves et al., 2010; Ribeiro et al., 2020) e medicinais (Ojewole, 2005; Remila et al., 2015).

\section{Considerações Finais}

O Japão e Estados Unidos da América destacam-se como os maiores detentores de pedidos de patentes registradas envolvendo a família, majoritariamente registrados a partir da década de 2010. Destaca-se que o Brasil pela sua grande biodiversidade possui potencial para produzir tecnologias em grande escala.

O Brasil é o país que mais publica pesquisas relacionadas a família, seguido de Estados Unidos e Índia. Houve o crescimento de publicações científicas sobre o grupo, nos últimos 20 anos. As pesquisas tiveram como principal objetivo o uso das espécies para finalidade econômica e farmacêutica incluindo principalmente atividade citotóxica e antibacteriana.

Considera-se importante que o país realize investimentos em pesquisas científicas para aprofundar os conhecimentos sobre propriedades medicinais de plantas do grupo, melhoramento de culturas para a cadeia produtiva. Além disto, faz-se necessário o investimento e desburocratização na produção e patenteamento de tecnologias com insumos advindos da biodiversidade, para que o Brasil melhorar sua posição no ranking dos países que mais produzem tecnologias com espécies de Anacardiaceae.

Pela vasta ocorrência de espécies dessa família no Brasil, apresenta-se como promissora para avanços farmacológico, medicinais e econômicos. Entretanto, o número de pedidos de patentes ainda é pequeno e demonstram que existe a necessidade de explorar estudos voltados para as atividades biológicas, bioprospecção de moléculas e importância econômica. Além disto, que se façam mapeamentos tecnológicos e científicos, a fim de expandir e complementar os conhecimentos sobre as espécies de Anacardiaceae.

\section{Referências}

Alencar, S. R.; Silva, M. A. P.; Macedo, M. S.; Ribeiro, D. A.; Santos, M. A. F. \& Costa, N. C. (2016). Phytotoxic effect of Mangifera indica L. (Anacardiaceae) at different times of collection. Iheringia, Série Botânica, 71 (2), 175-183.

Albuquerque, H.; Dias, J.; Dantas, R.; Cabral, I.; Medeiros, A. \& Santos, V. (2015). Potencial atividade antiulcerogênica da espécie Spondias mombin. Journal of Biology \& Pharmacy and Agricultural Management, 10 (2), 1-8.

Araújo, W. B. C.; Campos, R. T. \& Campos, K. C. (2019). Análise da cadeia produtiva da manga em Petrolina. Revista de Política Agrícola, 27 (4), 122.

Barrett, C. F.; Lambert, J.; Santee, M. V.; Sinn, B. T.; Skibicki, S. V.; Stephens, H. M. \& Thixton, H. (2021). Genetic, morphological, and niche variation in the widely hybridizing Rhus integrifolia-Rhus ovata species complex. Plant Species Biology, 36 (1), 17-35.

Berlinck, R. G.; Borges, W. D. S.; Scotti, M. T. \& Vieira, P. C. (2017). A química de produtos naturais do Brasil do século XXI. Química Nova, 40 ( ), 706710 .

Bordignon, S. A. D. L. \& Ely, C. V. (2020). Schinus pampeana (Anacardiaceae): A New endangered species from Rio Grande do Sul, Brazil. Phytotaxa, 452 (4), 278-287.

Bolzani, V. D. S. (2016). Biodiversidade, bioprospecção e inovação no Brasil. Ciência e Cultura, 68 (1), 04-05.

Broinizi, P. R. B.; Andrade-Wartha, E. R. S. D.; Silva, A. M. D. O.; Novoa, A. J. V.; Torres, R. P.; Azeredo, H. M. C., \& Mancini-Filho, J. (2007). Avaliação da atividade antioxidante dos compostos fenólicos naturalmente presentes em subprodutos do pseudofruto de caju (Anacardium occidentale L.). Food Science and Technology, 27, 902-908.

Chaves, M. H.; Lopes, A. M. G. C.; Lopes, J. A. D.; Costa, D. A. D.; Oliveira, C. A. A. D.; Costa, A. F. \& Brito Júnior, F. E. M. (2010). Fenóis totais, atividade antioxidante e constituintes químicos de extratos de Anacardium occidentale L., Anacardiaceae. Revista Brasileira de Farmacognosia, 20 (1), 106112 .

Correia, S. D. J.; David, J. P. \& David, J. M. (2006). Metabólitos secundários de espécies de Anacardiaceae. Química Nova, 29 (), $1287-1300$.

Cho, J. S.; Jang, B. K. \& Lee, C. H. (2020). Breaking combinational dormancy of Rhus javanica L. seeds in South Korea: Effect of mechanical scarification and cold-moist stratification. South African Journal of Botany, 133, 174-177.

Cruz, R. L.; Alencar, B. A. \& Silva, M. E. (2017). Ações de sustentabilidade em cadeias produtivas: uma pesquisa em relação à produção do caju e do coco. Revista Reuna, 22 (3), 1-18. 
De Araújo, S.; Sousa, I. J. O.; Gonçalves, R. L. G.; Sousa França, A. R.; Santos Negreiros, P.; Silva Brito, A. K. \& Sousa Lima, E. B. (2018). Aplicações Farmacológicas e Tecnológicas da Goma do Cajueiro (Anacardium Occidentale L.)-um Produto Obtido da Flora Brasileira. Revista Geintec-gestao Inovacao e Tecnologias, 8 (1), 4292-4305.

De Carvalho, T. V.; Nascimento, M. G. P.; Bittencourt, C. B. \& Andrade, I. M. (2020). Prospecção Científica e Tecnológica de Malpighia emarginata DC.(Malpighiaceae): espécie economicamente importante do Brasil. Cadernos de Prospecção, 13 (3), 862.

Da Silva Oliveira, F. G.; Araújo, A. D.; Silva Almeida, J. R. G.; Silva, M. V. \& Santos Correia, M. T. (2020). Prospecção Tecnológica de Spondias tuberosa (Anacardiaceae). Revista Geintec-gestao Inovacao e Tecnologias, 10 (3), 5546-5552.

Da Silva, A. E. (2015). Análise fitoquímica das cascas do caule do cajueiro (Anacardium occidentale L.-Anacardiaceae). Estação Científica (UNIFAP), 3 (2), 81-88.

Fyfe, S.; Smyth, H. E.; Schirra, H. J.; Rychlik, M. \& Sultanbawa, Y. (2020). The Nutritional Potential of the Native Australian Green Plum (Buchanania obovata) Compared to Other Anacardiaceae Fruit and Nuts. Frontiers in Nutrition, 7, 311.

Gurjão, F. F.; Carneiro, G. G.; Pessoa, T.; Silva, D. R. S. \& Pê, P. R. (2015). Comportamento reológico de iogurte de cajá comercializado em Campina Grande, Paraíba. Revista Verde de Agroecologia e Desenvolvimento Sustentável, 10 (2), 39.

Hall, C. F.; \& Gil, A. D. S. B. (2017). Flora das cangas da Serra dos Carajás, Pará, Brasil: Anacardiaceae. Rodriguésia, 68, 911-916.

Harsono, T. \& Pasaribu, N. (2016). Diversity of gandaria (Bouea) based on morphological characters in Indonesia. Sabrao Journal of Breeding and Genetics, $48(04), 491-503$.

INPI - Instituto Nacional da Propriedade Industrial. (2021). Informação tecnológica: Busca de patentes. http://www.inpi.gov.br/menuservicos/informacao/busca-depatentes.

Jacobsen, R. H. F.; Silva, E. A.; Silveira, I. P.; Rolim, E. L. G.; Silva, L. K. \& Ribeiro, S. B. (2018). Cadeia produtiva da produção de suco natural na agricultura familiar: Estudo de caso suco tropical. Revista Brasileira de Ciências da Amazônia/Brazilian Journal of Science of the Amazon, 7 (2), 1-8.

Láscaris, M. P. S.; Chaves, A. C. S. D.; Leite, J. V.; Gonçalves, J. L. C. \& Nunes, T. P. (2019). Determinação de compostos bioativos, atividade antioxidante e antimicrobiana do extrato da fibra da manga (Mangifera indica L. cv. Tommy Aktins). Higiene alimentar,1 (1) $2764-2768$.

Li, Q.; Wang, X.; Chen, J.; Liu, C.; Li, T.; McClements, D. J. \& Liu, J. (2016). Antioxidant activity of proanthocyanidins-rich fractions from Choerospondias axillaris peels using a combination of chemical-based methods and cellular-based assay. Food chemistry, 208, 309-317.

Martinelli, G. \& Moraes, M. A. (2013). Livro vermelho da flora do Brasil. http://cncflora.jbrj.gov.br/arquivos/arquivos/pdfs/LivroVerme lho.pdf.

Monte, D. D. C.; Lopes, D. B. \& Contini, E. (2017). China: Nova potência também no agronegócio. Revista de Política Agrícola, 26 (3), 107-123.

Monteiro, A. S.; Rodrigues, R. C. E.; Silva, G. F. \& Albuquerque, P. M. (2017). Estudo da atividade antimicrobiana da casca da castanha de caju (Anacardium occidentale). The Journal of Engineering and Exact Sciences, 3 (4), 0705-0710.

Morishige, H. (1940). Investigação sobre os cristais de albumina em látex de Schinus terebinthifolius (Anacardiaceae). The Japanese Forest Society, 22 (4), $220-222$.

Muellner-Riehl, A. N.; Weeks, A.; Clayton, J. W; Buerki, S.; Nauheimer, L.; Chiang, Y. C. \& Pell, S. K. (2016). Molecular phylogenetics and molecular clock dating of Sapindales based on plastid rbcL, atpB and trnL-trnF DNA sequences. Taxon, 65 (5), 1019-1036.

Nagel, A.; Conrad, J.; Leitenberger, M.; Carle, R. \& Neidhart, S. (2016). Structural studies of the arabinogalactans in Mangifera indica L. fruit exudate. Food Hydrocolloids, 61 (1), 555-566.

Nunes, E. M.; França, A. R. M.; Silva, M. R. F.; Sá, V. C. \& Gurgel, I. A. (2018). Arranjos produtivos em dinâmicas de Desenvolvimento Regional: a cadeia da cajucultura no Território Açu-Mossoró (RN). Redes. Revista do Desenvolvimento Regional, 23 (2), 187-209.

Ojewole, J. A. O. (2005). Antiinflammatory, analgesic and hypoglycemic effects of Mangifera indica Linn.(Anacardiaceae) stem-bark aqueous extract. Methods and findings in experimental and clinical pharmacology, 27(8), 547-554.

Oliveira, C. C.; Lopes, T. D. V. C.; Monteiro, G. C. \& Lima, G. P. P. (2018). Caracterização de umbu (Spondia tuberosa) durante seu desenvolvimento. Revista Iberoamericana de Tecnología Postcosecha, 19 (2), 1-9.

Oliveira, D. A.; Moraes, J. A. A. \& Carvalho, J. L. M. (2017). Fatores determinantes na mudança do padrão de produção e consumo da manga no mercado nacional. Revista em Agronegócio e Meio Ambiente, 10 (1), 51-73.

OMS - Organização Mundial da Saúde. (2018). Global Innovation Index 2018. https://www.wipo.int/pressroom/en/ar ticles/2018/article_0005.html.

Pell, S. K. (2011). The families and genera of vascular plants. X. Flowering plants. Eudicots. Sapindales, Cucurbitales, 1 (1), 7-50.

Pimentel, V.; Vieira, V. A. M.; Mitidieri, T. L.; Oliveira, F. F. S. \& Pieroni, J. P. (2015). Biodiversidade brasileira como fonte da inovação farmacêutica: uma nova esperança? Revista do BNDES, 43 (1), 41-89.

Remila, S.; Atmani-Kilani, D.; Delemasure, S.; Connat, J. L.; Azib, L.; Richard, T. \& Atmani, D. (2015). Antioxidant, cytoprotective, anti-inflammatory and anticancer activities of Pistacia lentiscus (Anacardiaceae) leaf and fruit extracts. European Journal of Integrative Medicine, 7 (3), $274-286$.

Ribeiro, A. D.; Júnior, E. C. F.; Júnior, J. G. R.; Costa, B. P.; Freire, J. C. P.; Souza Melo, W. O. \& Pereira, J. V. (2020). Potencial antimicrobiano do Anacardium occidentale Lin. contra patógenos orais. Research, Society and Development, 9 (8), e883986459-e883986459. 
Research, Society and Development, v. 10, n. 12, e408101220746, 2021

(CC BY 4.0) | ISSN 2525-3409 | DOI: http://dx.doi.org/10.33448/rsd-v10i12.20746

Ribeiro, F. D. O. S.; França Dourado, F.; Silva, M. F. S.; Brito, L. M.; Pessoa, C.; Lima, L. R. M. \& Silva, D. A. (2020). Anti-proliferative profile of Anacardium occidentale polysaccharide and characterization by AFM. International journal of biological macromolecules, 156, 981-987.

Salesse, D.; Medeiros, F. C.; Silva, C. C. M.; Lourenço, E. L. B. \& Jacomassi, E. (2018). Etnobotânica e etnofarmacologia das espécies de Amaryllidaceae, Anacardiaceae, Annonaceae e Apiaceae. Arquivos de Ciências da Saúde da UNIPAR, 22 (3), 1-12.

Santos, R.; Santos, R. \& Marisco, G. (2017). Avaliação da atividade genotóxica, citotóxica e antimicrobiana da infusão das folhas de Spondias purpurea L. Scientia Plena, 13 (3) 1-9.

Santos, N. A.; Gonçalves, J. F.; Oliveira, E. A. F.; Lucena, T. C., \& Sousa, R. M. (2018). Produção da Castanha de Caju nas microrregiões do Ceará no período de 1993 a 2016. Revista Eletrônica Competências Digitais para Agricultura Familiar, 4 (1), 103-116.

Santos, J. A. S.; Sena, T. J. O.; Costa, M. L. A.; Santos, K. C. B. S. \& Santos, A. F. (2018). Estudo do potencial antioxidante da Anacardium occidentales L. e determinação de seus compostos fenólicos. Diversitas Journal, 3 (2), 455-474.

Saraiva, A. M.; Castro, R. H.; Cordeiro, R. P.; Sobrinho, T. J. P.; Castro, V. T.; Amorim, E. L. \& Pisciottano, M. N. (2011). In vitro evaluation of antioxidant, antimicrobial and toxicity properties of extracts of Schinopsis brasiliensis Engl. (Anacardiaceae). African Journal of Pharmacy and Pharmacology, 5 (14), $1724-1731$.

Souza, V. C. \& Lorenzi, H. (2005). Botânica sistemática: guia ilustrado para identificação das famílias de Angiospermas da flora brasileira, baseado em $A P G$ II. Instituto Plantarum.

Flora do Brasil 2020 em construção. (2021). <http://floradobrasil.jbrj.gov.br/reflora/floradobrasil/FB44>.

Serafini, M. R..; Quintans, J. D. S. S.; Antoniolli, A. R.; Santos, M. R. V. \& Quintans-Junior, L. J. (2012). Mapeamento de tecnologias patenteáveis com o uso da hecogenina. Revista Geintec-gestao Inovacao e Tecnologias, 2 (5), 427-435.

Tavares, M. C.; Tölke, E. D.; Nunes, C. E. P. \& Carmello-Guerreiro, S. M. (2020). Floral morpho-anatomy and reproductive ecology of Spondias macrocarpa Engl. (Anacardiaceae), a vulnerable neotropical andromonoecious tree. Flora, 273, 151707.

Uchôa, A. D.; Oliveira, W. F.; Pereira, A. P.; Silva, A. G.; Cordeiro, B. M.; Malafaia, C. B., \& Correia, M. (2015). Antioxidant activity and phytochemical profile of Spondias tuberosa Arruda leaves extracts. American Journal of Plant Sciences, 6 (19), 3038-3044.

Yang, Y. Y.; Meng, Y.; Wen, J.; Sun, H. \& Nie, Z. L. (2016). Phylogenetic analyses of Searsia (Anacardiaceae) from eastern Asia and its biogeographic disjunction with its African relatives. South African Journal of Botany, 106, 129-136.

Zeraik, M. L.; Queiroz, E. F.; Marcourt, L.; Ciclet, O.; Castro-Gamboa, I.; Silva, D. H. S., \& Wolfender, J. L. (2016). Spondias tuberosa Antioxidants, quinone reductase inducers and acetylcholinesterase inhibitors from fruits. Journal of Functional foods, 21 (1), 396-405. 